\title{
РАСПОЗНАВАНИЕ СЕПСИСА В ОТДЕЛЕНИИ ИНТЕНСИВНОЙ ТЕРАПИИ НОВОРОЖДЕННЫХ С ИСПОЛЬЗОВАНИЕМ ИСКУССТВЕННОЙ НЕЙРОННОЙ СЕТИ
}

\section{SEPSIS RECOGNITION IN THE DEPARTMENT OF INTENSIVE NEWBORN THERAPY USING AN ARTIFICIAL NEURAL NETWORK}

Hassanin Hatem Mohamed Abdel Maksoud

Summary. Sepsis is the leading cause of morbidity and mortality worldwide. Early detection of sepsis is important because it allows for timely prescribing of potentially life-saving resuscitation and antimicrobial therapy [1].

Sepsis is a person's unregulated response to an infection that causes life-threatening organ dysfunction. According to statistics, [2] approximately one in three deaths in hospitals are associated with sepsis. Although effective protocols exist for the treatment of sepsis, problems remain with early and reliable detection of the condition [3]. In recent years, the increased adoption of electronic health records (EHRs) in hospitals has spurred the development of machine learning surveillance tools for detecting [4] and predicting [5] sepsis. However, most of the existing published models for predicting sepsis are based either on data from a single hospital [6] or from several hospitals in the same health care system where care processes are largely standardized. However, it is noted that the recognition of sepsis in the neonatal nonintensive care unit is practically not considered in the literature.

Sepsis is not limited to the intensive care unit. With advances in technology and data granularity that underlie clinical informatics systems, it is now possible to consider the development and implementation of sepsis alert systems in intensive care units and beyond. The rationale for using electronic sepsis surveillance is ultimately to facilitate timely and error-free treatment through early recognition and decision support. However, numerous barriers prevent the development and implementation of hospital-wide sepsis alert systems [7].

The aim of the presented study is to analyze methods for recognizing sepsis in the neonatal intensive care unit using an artificial neural network by analyzing the available electronic data of a medical record.

Keywords: sepsis, intensive care, artificial neural network, Convolutional neural network, Autoencoder.

\author{
Хассанин Хатем Мохамед Абдель Максуд \\ Аспирант, Начиональный исследовательский \\ Томский политехнический университет \\ Blombygg1@gmail.com
}

Аннотация. Сепсис - ведущая причина заболеваемости и смертности во всем мире. Раннее выявление сепсиса важно, поскольку оно позволяет своевременно назначить потенциально жизненно важную реанимацию и антимикробную терапию[1]. По статистике,[2] примерно одна из трех смертей в больницах связана с сепсисом.

Хотя существуют эффективные протоколы лечения сепсиса, остаются проблемы с ранним и надежным выявлением этого состояния[3]. В последние годы более широкое распространение электронных медицинских карт (ЭМК) в больницах послужило стимулом для разработки инструментов наблюдения на основе машинного обучения для обнаружения[4] и прогнозирования[5] сепсиса. Однако большинство существующих опубликованных моделей прогнозирования сепсиса основаны либо на данных одной больницы[6] либо на нескольких больницах той же системы здравоохранения где процессы ухода в основном стандартизированы. Однако отмечается, что распознавание сепсиса в отделении неинтенсивной терапии новорожденных в литературе практически не рассматривается.

Сепсис не ограничивается отделением интенсивной терапии. Благодаря достижениям в технологиях и детализации данных, лежащих в основе систем клинической информатики, теперь стало возможным рассмотреть вопрос о разработке и внедрении систем оповещения о сепсисе в отделениях интенсивной терапии и за их пределами. Причина использования электронного эпиднадзора за сепсисом состоит в том, чтобы в конечном итоге облегчить своевременное и безошибочное лечение за счет раннего распознавания и поддержки принятия решений. Однако многочисленные препятствия мешают разработке и внедрению систем оповещения о сепсисе в масштабах всей больницы[7].

Целью представленного исследования является анализ методов распознавания сепсиса в отделении интенсивной терапии новорожденных с использованием искусственной нейронной сети путем анализа доступных электронных данных медицинской карты.

Ключевые слова: сепсис, интенсивной терапии, искусственной нейронной сети, Сверточная нейронная сеть, Автоэнкодер. 
$\mathbf{P}$ азработка этих систем раннего оповещения вызвала значительный интерес к тому, как наилучшим образом использовать электронные данные для поиска и лечения пациентов в критическом состоянии,[8] а также заложить основу для внедрения систем оповещения о сепсисе в отделениях интенсивной терапии. Сложные технологии и все большие объемы данных в настоящее время поступают в существующие несовершенные системы электронных медицинских карт (ЭМИ) в учреждениях неотложной помощи. Этого неизбежного применения «больших данных» в здравоохранении нельзя и не следует избегать[9].

Однако разработка и внедрение систем оповещения о сепсисе без учета роста больших данных в клинической практике может привести к человеческим ошибкам[10], и информационной перегрузке[11].

Успешные системы оповещения о сепсисе должны быть разработаны таким образом, чтобы свести к минимуму утомление от предупреждений, прерывание, человеческий фактор и информационную перегрузку. Основная цель любой системы оповещения должна заключаться в том, чтобы вызвать реакцию медицинского работника в отделении интенсивной терапии, которая в противном случае не произошла бы или была бы отложена. Как и следовало ожидать, то, что медицинский работник делает с предупреждением, оказывает огромное влияние на эффективность автоматизированной системы предупреждений. Изменчивость в доступности ресурсов, вовлеченности руководства и заинтересованности клинических сторон оказывает фундаментальное влияние на то, окажут ли оповещения предполагаемое влияние на результаты[12].

Часто критическим шагом, ограничивающим частоту для эффективности систем оповещения о сепсисе, является доступность данных в реальном времени. Данные должны быть записаны, прежде чем алгоритм сможет их «увидеть» и сделать прогноз относительно того, подвержен ли пациент риску сепсиса или нет. Задержка ввода или проверки данных, отсутствие взаимосвязи систем и нечастое время выборки - все это способствует неоднородности, отсутствию или значительной задержке доступности данных в системах обнаружения сепсиса[13].

Напротив, клинический диагноз сепсиса часто основывается на суждениях и измерениях, которые нелегко зафиксировать. Эти измерения могут варьироваться от того, что новорожденный «плохо выглядит», увеличенное время наполнения капилляров, холодные пальцы ног, ограниченный пульс или повышенная работа дыхания. В настоящее время клинические алгоритмы выявления сепсиса не учитывают многие сигналы, которые врач принимает как должное. В результате, с точки зрения клинициста, предупреждения об обнаружении сепсиса часто срабатывают «поздно». Поздние оповещения представляют собой неприятные оповещения и значительно снижают эффективность системы оповещений.

Таким образом, системы оповещения о сепсисе будущего должны быть более точными. Передовое математическое моделирование и сложные методы машинного обучения могут быть использованы для обнаружения сепсиса у новорожденных[14].

Алгоритмы машинного обучения уже используются для классификации активности с помощью акселерометров на теле. Таким образом, актуально использование методов машинного обучения в системах оповещения о сепсисе для улучшения клинического ведения и лечения сепсиса[15].

Ветвь машинного обучения, нейронные сети, также известные как искусственные нейронные сети, представляют собой вычислительные модели - по сути, алгоритмы. Нейронные сети обладают уникальной способностью извлекать значение из неточных или сложных данных, чтобы находить закономерности и обнаруживать тенденции, слишком запутанные для человеческого мозга или других компьютерных технологий. Нейронные сети предоставили большее удобство различными способами[16].

Самым новаторским аспектом нейронных сетей является то, что после обучения они обучаются самостоятельно. Таким образом, они имитируют человеческий мозг, который состоит из нейронов, основного строительного блока для передачи информации как человеком, так и нейронными сетями. Нейронные сети обладают следующими атрибутами, которые могут быть полезны при разработке автоматизированной системы распознавания сепсиса в отделении интенсивной терапии новорожденных[17]:

- Адаптивное обучение.

- Самоорганизация.

- Работа в реальном времени.

- Прогноз.

- Отказоустойчивость.

Основные алгоритмы нейронных сетей, которые могут быть использованы для распознавания сепсиса, сведены в таблицу 1[18,19,20].

Важно определить, на основании каких данных, представленных в электронной медицинской карте, можно распознать сепсис (таблица 2). 


\section{Таблица 1. Основные алгоритмы обучения нейронных сетей}

\begin{tabular}{|c|c|}
\hline Алгоритм & Цель \\
\hline Автоэнкодер (АЕ) & $\begin{array}{l}\text { Обычно АЕ используется для уменьшения количества рассматриваемых случайных величин, } \\
\text { чтобы система могла изучить представление для набора данных и, следовательно, обработать } \\
\text { генеративные модели данных. }\end{array}$ \\
\hline $\begin{array}{l}\text { Двунаправленная рекуррентная } \\
\text { нейронная сеть (BRNN) }\end{array}$ & $\begin{array}{l}\text { Цель BRNN - увеличить информационные входы, доступные для сети, путем подключения двух } \\
\text { скрытых, направленных противоположных слоев к одному и тому же выходу. Используя BRNN, } \\
\text { выходной слой может получать информацию как из прошлого, так и из будущего состояния. }\end{array}$ \\
\hline Машина Больцмана (BM) & $\begin{array}{l}\text { Рекуррентная нейронная сеть, этот алгоритм способен изучать внутренние представления } \\
\text { и может представлять и решать сложные комбинированные задачи. }\end{array}$ \\
\hline $\begin{array}{l}\text { Сверточная нейронная сеть } \\
\text { (CNN) }\end{array}$ & $\begin{array}{l}\text { CNN, наиболее часто используемые для анализа визуальных образов, представляют собой } \\
\text { нейронную сеть с прямой связью, предназначенную для минимизации предварительной } \\
\text { обработки. }\end{array}$ \\
\hline $\begin{array}{l}\text { Деконволюционная нейронная } \\
\text { сеть (DNN) }\end{array}$ & $\begin{array}{l}\text { DNN позволяют неконтролируемое построение иерархических представлений изображений. } \\
\text { Каждый уровень иерархии группирует информацию из предыдущего уровня для добавления } \\
\text { более сложных функций к изображению. }\end{array}$ \\
\hline Сеть глубокого убеждения (DBN) & $\begin{array}{l}\text { При обучении на неконтролируемом наборе примеров DBN может научиться } \\
\text { реконструировать свои входные данные вероятностно, используя слои в качестве детекторов } \\
\text { признаков. Следуя этому процессу, можно обучить DBN выполнять контролируемые } \\
\text { классификации. }\end{array}$ \\
\hline $\begin{array}{l}\text { Сеть глубокой сверточной } \\
\text { обратной графики (DCIGN) }\end{array}$ & $\begin{array}{l}\text { Модель DCIGN предназначена для изучения интерпретируемого представления, которые } \\
\text { система разделяет в соответствии с элементами трехмерной структуры сцены, такими как } \\
\text { вариации освещения и поворот глубины. DCIGN использует множество уровней операторов, } \\
\text { как сверточных, так и деконволюционных. }\end{array}$ \\
\hline Глубокая остаточная сеть (DRN) & $\begin{array}{l}\text { DRN помогают в решении сложных задач и моделей глубокого обучения. Имея много уровней, } \\
\text { DRN предотвращает ухудшение результатов. }\end{array}$ \\
\hline $\begin{array}{l}\text { Автоэнкодер с шумоподавлением } \\
\text { (DAE) }\end{array}$ & $\begin{array}{l}\text { DAE используется для восстановления данных из поврежденных входных данных; алгоритм } \\
\text { заставляет скрытый слой изучать более надежные функции. В результате на выходе получается } \\
\text { более точная версия входных данных. }\end{array}$ \\
\hline Сеть состояния эха (ESN) & $\begin{array}{l}\text { ESN работает со случайной большой фиксированной рекуррентной нейронной сетью, } \\
\text { в которой каждый узел получает нелинейный ответный сигнал. Алгоритм случайным образом } \\
\text { устанавливает и назначает веса и возможности подключения для достижения гибкости } \\
\text { обучения. }\end{array}$ \\
\hline $\begin{array}{l}\text { Машина экстремального } \\
\text { обучения (ELM) }\end{array}$ & $\begin{array}{l}\text { Этот алгоритм изучает выходные веса скрытых узлов за один шаг, создавая линейную } \\
\text { модель. ЕLМ могут хорошо обобщать и учиться во много раз быстрее, чем сети обратного } \\
\text { распространения. }\end{array}$ \\
\hline $\begin{array}{l}\text { Нейронная сеть прямого } \\
\text { распространения (FF или FFNN) } \\
\text { и персептрон (P) }\end{array}$ & $\begin{array}{l}\text { Это базовые алгоритмы нейронных сетей. Нейронная сеть прямого распространения — это } \\
\text { искусственная нейронная сеть, в которой соединения узлов не образуют цикл; перцептрон - } \\
\text { это бинарная функция, имеющая только два результата (вверх / вниз; да / нет, 0/1). }\end{array}$ \\
\hline Закрытый рекуррентный блок & $\begin{array}{l}\text { ЗРБ используют соединения через последовательности узлов для выполнения задач } \\
\text { машинного обучения, связанных с кластеризацией и памятью. ЗРБ уточняют выходные данные } \\
\text { посредством управления информационным потоком модели. }\end{array}$ \\
\hline $\begin{array}{l}\text { Генеративная состязательная сеть } \\
\text { (GAN) }\end{array}$ & $\begin{array}{l}\text { Эта система противопоставляет две нейронные сети - дискриминационную и генеративную. } \\
\text { Цель состоит в том, чтобы различать реальные и синтетические результаты для моделирования } \\
\text { концептуальных задач высокого уровня. }\end{array}$ \\
\hline Сеть Хопфилда (HN) & $\begin{array}{l}\text { Эта форма рекуррентной искусственной нейронной сети представляет собой систему } \\
\text { ассоциативной памяти с бинарными пороговыми узлами. Созданные для сведения } \\
\text { к локальному минимуму, HN представляют собой модель для понимания человеческой памяти. }\end{array}$ \\
\hline Сеть Кохонена (KN) & $\begin{array}{l}\text { KN организует проблемное пространство в двумерную карту. Разница между } \\
\text { самоорганизующимися картами (SOM) и другими подходами к решению проблем заключается } \\
\text { в том, что SOM используют конкурентное обучение, а не обучение с исправлением ошибок. }\end{array}$ \\
\hline Машина жидких состояний (LSM) & $\begin{array}{l}\text { LSM, известный как машинное обучение третьего поколения (или импульсная нейронная } \\
\text { сеть), добавляет понятие времени как элемента. LSM генерируют активацию пространственно- } \\
\text { временной нейронной сети, поскольку они сохраняют память во время обработки. Физика } \\
\text { и вычислительная нейробиология используют LSM. }\end{array}$ \\
\hline
\end{tabular}


Таблица 1 (продолжение). Основные алгоритмы обучения нейронных сетей

\begin{tabular}{|c|c|}
\hline Алгоритм & Цель \\
\hline $\begin{array}{l}\text { Долговременная / } \\
\text { кратковременная память (LSTM) }\end{array}$ & $\begin{array}{l}\text { LSTM способен изучать или запоминать зависимость порядка в задачах прогнозирования, } \\
\text { касающихся последовательности. Блок LSTM содержит ячейку, входной вентиль, выходной } \\
\text { вентиль и вентиль забывания. Ячейки сохраняют значения в течение произвольных интервалов } \\
\text { времени. Каждый блок регулирует потоки значений через LSTM-соединения. Эта возможность } \\
\text { упорядочивания необходима в сложных проблемных областях, таких как распознавание. }\end{array}$ \\
\hline Цепь Маркова (МС) & $\begin{array}{l}\text { МС - это математический процесс, который описывает последовательность возможных } \\
\text { событий, в которой вероятность каждого события зависит исключительно от состояния, } \\
\text { достигнутого в предыдущем событии. }\end{array}$ \\
\hline $\begin{array}{l}\text { Нейронная машина Тьюринга } \\
\text { (NTM) }\end{array}$ & $\begin{array}{l}\text { Основываясь на работе специалиста по обработке данных Алана Тьюринга середины 20-го } \\
\text { века, NTM выполняет вычисления и расширяет возможности нейронных сетей за счет связи } \\
\text { с внешней памятью. Разработчики используют НТМ в роботах и рассматривают его как одно } \\
\text { из средств создания искусственного человеческого мозга. }\end{array}$ \\
\hline $\begin{array}{l}\text { Сети радиальных базисных } \\
\text { функций (сети RBF) }\end{array}$ & $\begin{array}{l}\text { Разработчики используют сети RBF для моделирования данных, которые представляют } \\
\text { основную тенденцию или функцию. Сети RBF учатся аппроксимировать основной тренд, } \\
\text { используя кривые колокола или нелинейные классификаторы. Нелинейные классификаторы } \\
\text { анализируют более глубоко, чем простые линейные классификаторы, которые работают } \\
\text { с векторами меньшей размерности. Вы используете эти сети в управлении системой } \\
\text { и прогнозировании временных рядов. }\end{array}$ \\
\hline $\begin{array}{l}\text { Рекуррентная нейронная сеть } \\
\text { (RNN) }\end{array}$ & $\begin{array}{l}\text { PHC моделируют последовательные взаимодействия через память. На каждом временном } \\
\text { шаге RNN вычисляет новую память или скрытое состояние в зависимости от текущего входного } \\
\text { и предыдущего состояния памяти. Приложения включают сочинение музыки, управление } \\
\text { роботом и распознавание действий человека. }\end{array}$ \\
\hline $\begin{array}{l}\text { Ограниченная машина Больцмана } \\
\text { (RBM) }\end{array}$ & $\begin{array}{l}\text { RBM - это вероятностная графическая модель в неконтролируемой среде. RBM состоит } \\
\text { из видимых и скрытых слоев, а также связей между бинарными нейронами в каждом из этих } \\
\text { слоев. RBN полезны для фильтрации, изучения функций и классификации. }\end{array}$ \\
\hline Машина опорных векторов (SVM) & $\begin{array}{l}\text { На основе наборов обучающих примеров, относящихся к одной из двух возможных категорий, } \\
\text { алгоритм SVM строит модель, которая относит новые примеры к одной из двух категорий. } \\
\text { Затем модель представляет примеры в виде нанесенных на карту точек в пространстве, при } \\
\text { этом эти примеры отдельных категорий делятся на максимально возможный промежуток. }\end{array}$ \\
\hline
\end{tabular}

Таблица 2

\begin{tabular}{|l|l|l|}
\hline - Плохое сосание; & -Апноэ; & - Сниженную спонтанную активность; \\
\hline - Анорексию; & - Брадикардию; & $\begin{array}{l}\text { - Колебания температуры тела } \\
\text { (гипотермия или гипертермия)[21]. }\end{array}$ \\
\hline
\end{tabular}

Особенно частые ранние признаки сепсиса включают в себя следующие:

Лихорадка возникает только в 10-15\% случаев, однако упорная лихорадка (например, >1 ч) обычно указывает на наличие инфекции.

Другие симптомы и признаки включают дыхательную недостаточность, неврологические симптомы (например, судороги, повышенную нервную возбудимость), желтуху (особенно возникающую в течение первых 24 ч жизни при отсутствии несовместимости по группам крови систем Rh или ABO и с более высокой, чем ожидалось, концентрацией прямого билирубина), рвоту, диарею и вздутие живота.
Все эти данные могут быть отражены в электронной медицинской карте. Помимо этого, выполняются анализы, которые дополнительно позволяют выявить сепсис.

Отмечается, что новорожденным с клиническими признаками сепсиса как можно скорее необходимо провести общий анализ крови, дифференциальный анализ с помощью мазка, посев крови и мочи и люмбальную пункцию (ЛП), если клинически осуществимо[22].

Лабораторные признаки, которые помогут выявить сепсис у новорожденных:

- Лейкоцитоз > 15×10\%;

- Лейкопения 9/л; 


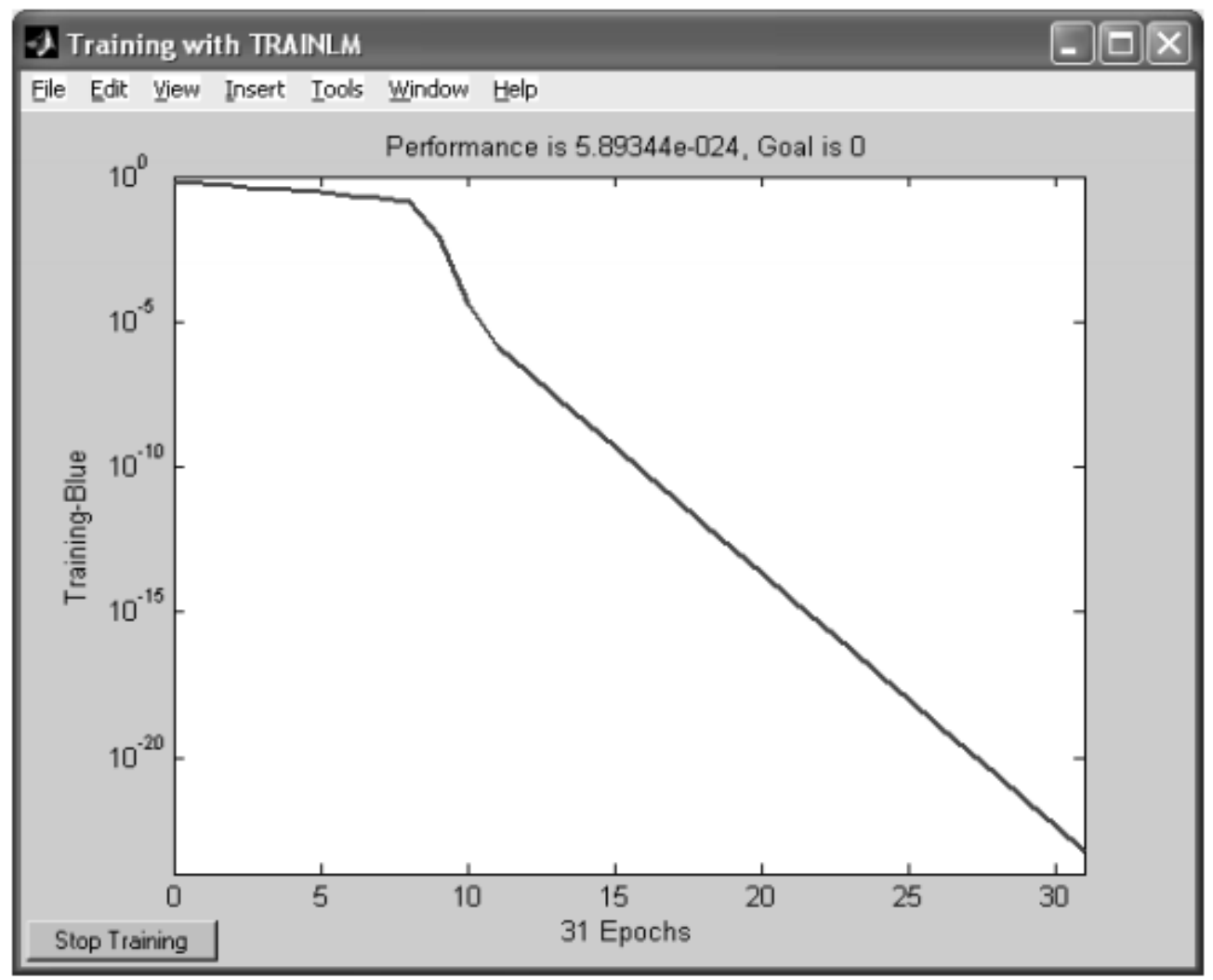

Рис. 1. Процесс обучения НС

A Neural Fitting (nftool)

\section{Network Architecture}

- Set the number of neurons in the fitting network's hidden layer. Hidden Layer

Define a fitting neural network. (fitnet)

Number of Hidden Neurons:

Recommendation

Return to this panel and change the number of neurons if the network does not perform well after training.

\section{Restore Defaults}

Neural Network

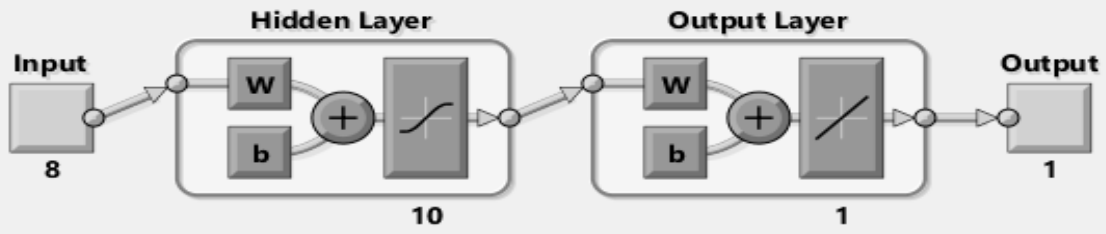

Рис. 2. Структура нейронной сети для распознавания сепсиса у новорожденных 


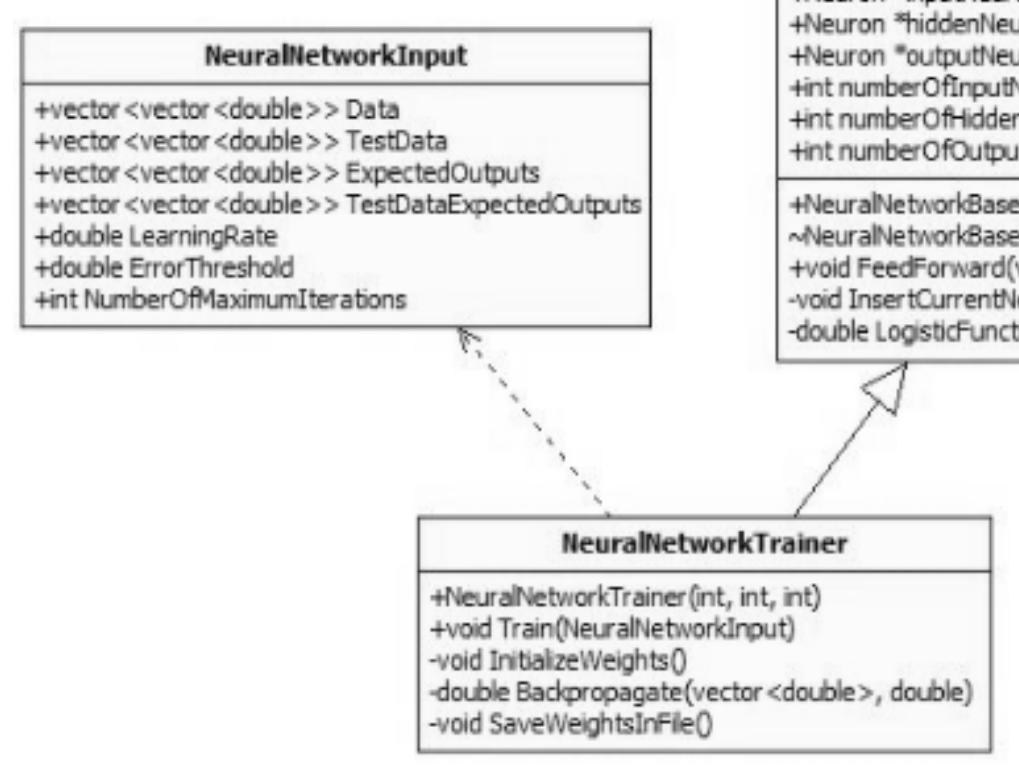

\begin{tabular}{|l|l|}
\hline \multicolumn{1}{|c|}{ NeuralNetworkBase } \\
\hline $\begin{array}{l}\text { +Neuron *inputNeurons } \\
\text { +Neuron *hiddenNeurons } \\
\text { tNeuron "outputNeurons } \\
\text { tint numberOfinputNeurons } \\
\text { tint numberOfHiddenNeurons } \\
\text { tint numberOfOutputNeurons }\end{array}$ \\
\hline $\begin{array}{l}\text { +NeuralNetworkBase(int, int, int) } \\
\text { MeuralNetworkBase0 }\end{array}$
\end{tabular}

Рис. 3. Диаграмма классов, перераспределенная в процессе обучения нейронной сетью на электронных медицинских картах

\section{1- Network Architecture}

1 Choose the number of neurons and input/feedback delays.

Architecture Choices

Define a NARX neural network. (narxnet)

Number of Hidden Neurons:

10

Number of delays d:

2

Problem definition:

Recommendation

Return to this panel and change the number of neurons or delays if the network does not perform well after training.

The network will be created and trained in open loop form as shown below. Open loop (single-step) is more efficient than closed loop (multi-step) training. Open loop allows us to supply the network with correct past outputs as we train it to produce the correct current outputs.

After training, the network may be converted to closed loop form, or any other form, that the application requires.

\section{Restore Defaults}

Neural Network

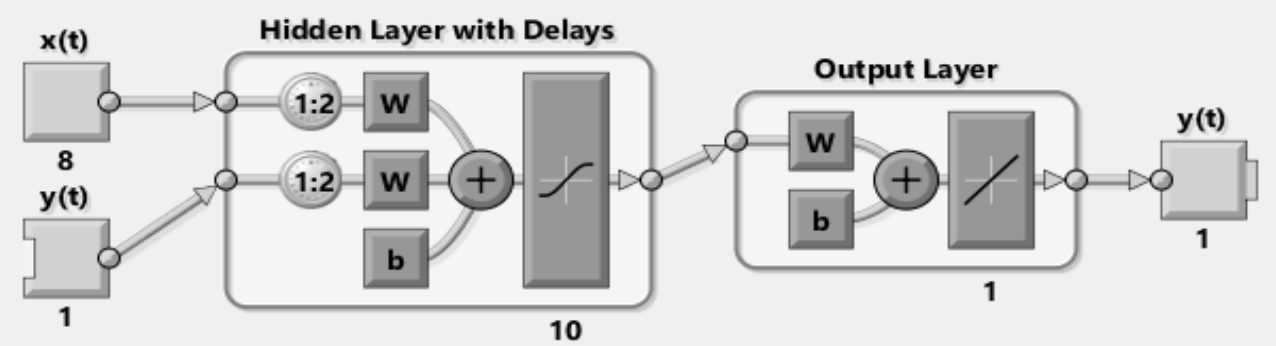

Рис. 4. Прогнозная модель распознавания сепсиса в MatLab 
- Нейтрофилез > 6×10\%/л;

Нейтропения $9 / л$

Юные формы нейтрофилов > 1,5×10\%/л;

Токсическая зернистость нейтрофилов;

Нейтрофильный индекс >0,2;

Уровень СР-белка > 6 мг/л;

Уровень прокальцитонина > 2 нг/мл;

Уровень интерлейкина-8 > 100 пг/мл[23].

Данная информация поможет нейронной сети распознать сепсис у новорожденного. Искусственные нейронные сети в среде MATLAB можно проектировать как с помощью специального встроенного пакета NNTool, так и непосредственно в командном окне. С помощью программы можно изображать процесс обучения для нейронных сетей (рисунок 1) в виде графика, а значит, пользователь может определить, как проходил процесс обучения и сколько циклов обучения понадобилось, чтобы ошибка обучающего множества достигла нужного уровня.

Структура нейронной сети для распознавания сепсиса у новорожденных представлена на рисунке 2

На рисунке 3 представлена диаграмма классов, перераспределенная в процессе обучения.

Предлагаемая сеть состоит из трех слоев: входной слой, скрытый слой, и выходной слой. В качестве обучающей выборки могут быть собраны обезличенные данные электронных медицинских карт новорожденных.

Прогнозная модель распознавания сепсиса представлена на рисунке 4.

На рисунке 4 представлены:

w - Вес входящего показателя для построения нейронной сети; d - Количество задержек нейронной сети;

Hidden Layer Delays - скрытый слой обучения с задержками;

Output Layer - выходной слой.

Загрузка данных для обучения нейронной сети проводится непосредственно из медицинской карты. При этом используются следующие функции:

$A=$ importdata(filename),

$A=$ importdata('-pastespecial')

$A=\operatorname{importdata(\ldots ,}$, delimiterln),

$A=$ importdata (__, delimiterln, headerlines $I n)$

$[\mathrm{A}$, delimiterOut, headerlinesOut $]=$ importdata (

Как только нейронная сеть соответствует данным, она формирует обобщение отношения ввода-вывода и может использоваться для распознавания сепсиса для сведений неворожденных, на которых сеть не обучалась.

\section{Зак^ючение}

В статье рассмотрена структура нейронной сети для распознавания сепсиса в отделении интенсивной терапии новорожденных путем анализа доступных электронных данных медицинской карты. Учитывая текущую неспособность диагностировать сепсис у новорожденных, меры по контролю состояния должны вводиться эмпирически и деэскалироваться, если сепсис не выявлен. Хотя может показаться, что изначально эти факторы снижают потенциальную точность любой системы оповещения о сепсисе, глобальная осведомленность о сепсисе повысит точность этих систем. Рассмотренный подход с использованием нейронных сетей является основой для создания автоматизированных систем реагирования и распознавания сепсиса в отделениях интенсивной терапии.

\section{ЛИТЕРАТУРА}

1. Shashikumar, S.P., Wardi, G., Malhotra, A. et al. Artificial intelligence sepsis prediction algorithm learns to say “I don't know”. npj Digit. Med., 2021.- - 4, 134 p.

2. Rhee, C. et al. Incidence and trends of sepsis in US hospitals using clinical vs claims data, 2009-2014. JAMA 318, 2017, p. 1241-1249.

3. Mao, Q. et al. Multicentre validation of a sepsis prediction algorithm using only vital sign data in the emergency department, general ward and ICU. BMJ Open 8, e017833, 2018. - $12 \mathrm{p}$.

4. Desautels, T. et al. Prediction of sepsis in the intensive care unit with minimal electronic health record data: a machine learning approach. JMIR Med. Inform. 4, e28, 2016. - $25 \mathrm{p}$.

5. Reyna, M.A. et al. Early prediction of sepsis from clinical data: the PhysioNet/Computing in Cardiology Challenge 2019. Crit. Care Med. 1, 2019.

6. Fleuren, L.M. et al. Machine learning for the prediction of sepsis: a systematic review and meta-analysis of diagnostic test accuracy. Intensive Care Med., 2020. - 25 p.

7. Sendak, M.P., Gao, M., Brajer, N. \& Balu, S. Presenting machine learning model information to clinical end users with model facts labels. NPJ Digital Med. 3, 2020, p. 1-4.

8. Singal G, Currier P. How can we best use electronic data to find and treat the critically ill?. Crit Care Med. 2012;40:2242-3.

9. Murdoch TB, Detsky AS. The inevitable application of big data to health care. JAMA. 2013; 309:1351-2. 
10. Herasevich V, Kor DJ, Subramanian A, et al. Connecting the dots: rule-based decision support systems in the modern EMR era. J Clin Monit Comput. 2013; 27(4):443-8.

11. Harrison AM, Herasevich V, Gajic 0. Automated sepsis detection, alert, and clinical decision support: act on it or silence the alarm? Crit Care Med. 2015 ; 43:1776-7.

12. Snoek, J., Larochelle, H. \& Adams, R.P. Practical bayesian optimization of machine learning algorithms. Adv. Neural Inf. Process. Syst., 2012.— p. $2951-2959$.

13. Moskowitz, A., McSparron, J., Stone, D.J. \& Celi, L.A. Preparing a new generation of clinicians for the era of big data. Harv. Med. Stud. 2015, Rev. 2, 24p.

14. Mannini A, Sabatini AM. Machine learning methods for classifying human physical activity from on-body accelerometers. Sensors (Basel). 2010; 10:1154-75.

15. Gill PS, Kamath A, Gill TS. Distraction: an assessment of smartphone usage in health care work settings. Risk Manag Healthc Policy. 2012; 5:105-14.

16. Барский, А.Б. Логические нейронные сети: Учебное пособие / А.Б. Барский. — М.: Бином, 2013.— 352 с.

17. Галушкин, А.И. Нейронные сети: основы теории. / А.И. Галушкин. — М.: РиС, 2015.— 496 с.

18. Zhang W., Zhang Z., Chao H.C., \& Guizani M. Toward Intelligent Network Optimization in Wireless Networking: An Auto-Learning Framework. IEEE Wireless Communications, 26(3), 2019, pp.76-82.

19. Arulkumaran K.; Deisenroth M.P.; Brundage M.; et al. Deep Reinforcement Learning: A Brief Survey. IEEE Signal Processing Magazine, $34(6), 2017$ pp. $26-38$.

20. Joy N.M.; Baskar M.K.; \& Umesh S. DNNs for unsupervised extraction of pseudo speaker-normalized features without explicit adaptation data. Speech Communication, 92, 2017, pp. 64-76.

21. Неонатальный сепсис [Электронный ресурс]: Режим доступа: https://www.msdmanuals.com/ru (дата обращения: 05.10.2021).

22. Ро0з Р., Генцель-Боровичеши 0., Прокитте Г. Неонатология. Практические рекомендации. — М.: Медицинская литература, 2011. - 568 c.

23. Сепсис новорожденных. Современные проблемы диагностики и лечения [Электронный ресурс]: Режим доступа: https://medi.ru/info/3430/ (дата 06ращения: 05.10.2021).

(с) Хассанин Хатем Мохамед Абдель Максуд ( Blombygg1@gmail.com ).

Журнал «Современная наука: актуальные проблемы теории и практики»

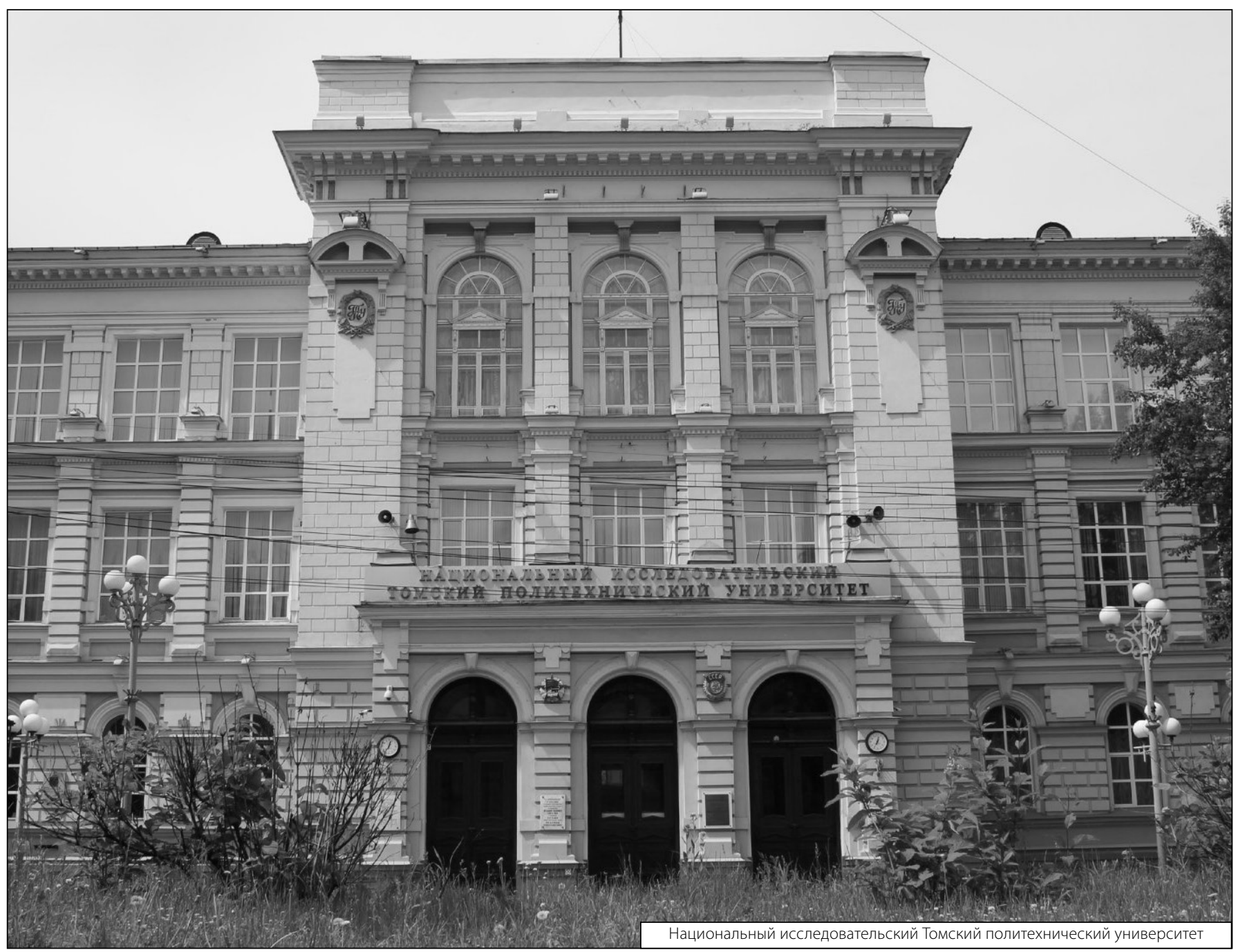

